Draft version February 17, 2021

Typeset using $\mathrm{LAT}_{\mathrm{E}} \mathrm{X}$ twocolumn style in AASTeX63

\title{
Matter density distribution of general relativistic highly magnetized jets driven by black holes
}

\author{
Taiki Ogihara, ${ }^{1}$ Takumi Ogawa, ${ }^{2,1}$ and Kenji Toma ${ }^{3,1}$ \\ ${ }^{1}$ Astronomical Institute, Graduate School of Science, Tohoku University, Sendai, Miyagi, 980-8578, Japan \\ ${ }^{2}$ Center for Computational Sciences, University of Tsukuba, Tsukuba, Ibaraki, 305-8577, Japan \\ ${ }^{3}$ Frontier Research Institute for Interdisciplinary Sciences, Tohoku University, Sendai, Miyagi, 980-8578, Japan
}

\begin{abstract}
High-resolution very long baseline interferometry (VLBI) radio observations have resolved the detailed emission structures of active galactic nucleus jets. General relativistic magnetohydrodynamic (GRMHD) simulations have improved the understanding of jet production physics, although theoretical studies still have difficulties in constraining the origin and distribution of jetted matter. We construct a new steady, axisymmetric GRMHD jet model to obtain approximate solutions of black hole $(\mathrm{BH})$ magnetospheres, and examine the matter density distribution of jets. By assuming fixed poloidal magnetic field shapes that mimic force-free analytic solutions and GRMHD simulation results and assuming constant poloidal velocity at the separation surface, which divides the inflow and outflow, we numerically solve the force-balance between the field lines at the separation surface and analytically solve the distributions of matter velocity and density along the field lines. We find that the densities at the separation surface in our parabolic field models roughly follow $\propto r_{\mathrm{ss}}^{-2}$ in the far zone from the $\mathrm{BH}$, where $r_{\mathrm{ss}}$ is the radius of the separation surface. When the $\mathrm{BH}$ spin is larger or the velocity at the separation surface is smaller, the density at the separation surface becomes concentrated more near the jet edge. Our semi-analytic model, combined with radiative transfer calculations, may help interpret the high-resolution VLBI observations and understand the origin of jetted matter.
\end{abstract}

Keywords: Active galactic nuclei (16), Black hole physics (159), General relativity (641), Relativistic jets (1390), Magnetohydrodynamics (1964)

\section{INTRODUCTION}

Relativistic collimated outflows (or jets) are observed in active galactic nuclei (AGNs). They originate from the system composed of a supermassive black hole (BH), an accretion disk, and a surrounding gas at the center of the galaxy. Very long baseline interferometry (VLBI) radio observations have revealed detailed emission structures of AGN jets. Limb-brightened structures have been observed at mm-cm wavelengths in jets of M87 (Kovalev et al. 2007; Walker et al. 2008; Hada et al. 2011, 2016; Mertens et al. 2016; Kim et al. 2018; Walker et al. 2018), Mrk 501 (Piner et al. 2008), Mrk 421 (Piner et al. 2010), Cyg A (Boccardi et al. 2016), and 3C84 (Nagai et al. 2014; Giovannini et al. 2018), and a triple-ridge structure composed of the limb-brightened components and an additional central bright component has been

Corresponding author: Taiki Ogihara

t.ogihara@astr.tohoku.ac.jp observed in the M87 jet (Asada et al. 2016; Hada et al. 2017; Walker et al. 2018). The Event Horizon Telescope (EHT) has revealed the ring-like emission structure just around the BH horizon of M87 (Event Horizon Telescope Collaboration et al. 2019a,b,c,d,e,f). These highresolution observations have provided us hints for testing theoretical jet models.

The plausible driving mechanism of relativistic jets is the Blandford-Znajek (BZ) process (Blandford \& Znajek 1977). The frame-dragging effect on a rotating $\mathrm{BH}$ magnetosphere twists the poloidal magnetic field lines that thread the ergosphere and creates the outward Poynting flux extracting the energy and angular momentum from the BH (Koide et al. 2002; Komissarov 2004; Dermer \& Menon 2009; Toma \& Takahara 2014, 2016; Kinoshita \& Igata 2018). General relativistic magnetohydrodynamic (GRMHD) simulations show that a highly magnetized region emerges around the rotational axis, where the BZ process works (e.g. McKinney \& Gammie 2004; McKinney \& Blandford 2009; Tchekhovskoy et al. 2011; Takahashi et al. 2016; Nakamura et al. 2018; Porth et al. 
2019). The Poynting flux can be converted to the matter kinetic energy flux, accelerating the matter to the relativistic speed (e.g. Komissarov et al. 2007; Komissarov et al. 2010; Lyubarsky 2009; Tchekhovskoy et al. 2009; Beskin 2010; Toma \& Takahara 2013; Tanaka \& Toma 2020).

A fundamental problem is the origin of the jetted matter. In the GRMHD simulations, the number density in the highly magnetized region becomes low around the 'separation surface' between the inflow passing through the $\mathrm{BH}$ horizon and the outflow accelerating relativistically. The continuous injection of plasma is required to maintain a steady jet, although the large-scale magnetic field lines prevent thermal plasma from diffusing into the highly magnetized region. Plasma could be replenished via the $e^{+} e^{-}$pair-creation gap formation (e.g. Blandford \& Znajek 1977; Beskin et al. 1992; Broderick \& Tchekhovskoy 2015; Hirotani et al. 2016; Levinson \& Segev 2017; Kisaka et al. 2020), and/or the annihilation of high-energy photons from the accretion disk (Levinson \& Rieger 2011; Mościbrodzka et al. 2011; Kimura \& Toma 2020). However, these non-thermal effects are not considered in GRMHD simulations because of uncertainties in the physics of mass loading. Instead, they set the artificial density floor values to prevent the numerical difficulty (McKinney \& Gammie 2004; Riordan et al. 2018).

Solving the steady equations do not need the density floor. The mass density distribution of the solutions may constrain the mass-loading mechanisms. The analytical solution of the steady axisymmetric GRMHD flow along a prescribed field line has been developed in the literature (Bekenstein \& Oron 1978; Camenzind 1986; Takahashi et al. 1990; Globus \& Levinson 2014). Once the field line configuration and the Bernoulli parameters are given, one can solve the Bernoulli equation (or wind equation) for each field line. Pu et al. (2015) showed an analytic solution for the single field line which mimics the GRMHD simulation results (Tchekhovskoy et al. 2010; Nakamura et al. 2018). Pu \& Takahashi (2020) presented a wind solution, which successfully pass the fast points, for multiple field lines with the prescribed toroidal field, but they do not consider the forcebalance between the field lines (or the Grad-Shafranov (GS) equation). To solve the GS equation is computationally demanding (Nitta et al. 1991; Fendt 1997; Beskin 2010; Nathanail \& Contopoulos 2014; Pan et al. 2017; Mahlmann et al. 2018). Recently, Huang et al. (2019) and Huang et al. (2020) succeeded in constructing steady axisymmetric numerical solutions for the monopole and parabolic field line configurations by iteratively solving the wind equation and the GS equa- tion. In Huang et al. (2020), they introduced the 'loading zone'. The inner boundary of the loading zone is the null-charge surface, and the outer boundary is the surface where the Bernoulli equation's solution of the outflow becomes $u_{\mathrm{p}}=0$. The outflow and inflow start at these surfaces. They assumed the fluid number flux per magnetic flux $\eta$ as constant for different field lines, and did not try to constrain the mass-loading mechanism.

One can also investigate the distribution of mass density as well as other physical quantities by comparing observations with the synthetic images derived by radiative transfer calculations in the jet models (Broderick \& Loeb 2009; Porth et al. 2011; Dexter et al. 2012; Lu et al. 2014; Mościbrodzka et al. 2014, 2016, 2017; Dexter 2016; Jiménez-Rosales \& Dexter 2018; Kawashima et al. 2019; Chael et al. 2018, 2019; Davelaar et al. 2019; Chatterjee et al. 2020; Jeter et al. 2020). Based on special relativistic force-free steady jet models, Takahashi et al. (2018) showed that a rapidly rotating $\mathrm{BH}$ accelerates the flow efficiently with remaining small toroidal velocity, leading to symmetric limb-brightened structure, which is compatible with the large-scale observations of M87 jet, and Ogihara et al. (2019) showed that the velocity field structure naturally produces the observed central ridge emission of M87 jet by relativistic beaming effect (see also Chernoglazov et al. 2019). They also demonstrated that the synthetic images of large-scale jets strongly depend on the density distribution of jets near the BHs. Kawashima et al. (2020) performed GR radiative transfer calculations to show that the emission at the bottom of the separation surface can reproduce the ring-like image just around the M87 BH. Future EHT observations of M87 will unveil the emission structure between the limb-brightened one and the ring-like image (Hada 2019). Parametric studies of steady GRMHD jet models and their comparison with the observations will extract the mass density distribution and other specific conditions around the separation surface.

In this paper, we introduce a new way to construct a steady axisymmetric GRMHD approximate solution to examine the jet's density distribution without being suffered from the density floor problem. We fix the field line configuration which mimics the ones of forcefree or highly-magnetized GRMHD simulation results (Tchekhovskoy et al. 2008, 2010; Nakamura et al. 2018; Porth et al. 2019) with an additional term for obtaining trans-fast-magnetosonic solutions (c.f. Beskin \& Nokhrina 2006; $\mathrm{Pu}$ et al. 2015). We numerically solve the transverse force-balance between the field lines at the separation surface to determine the distribution of the Bernoulli parameters, which include $\eta$, and analytically 
solve the Bernoulli equation along each field line. We assume the poloidal velocities at the separation surface as constant for different field lines, and our model does not employ the 'loading zone.' This paper is organized as follows. In Section 2, we outline governing equations of steady, axisymmetric, cold, ideal GRMHD flows and our method to obtain approximate solutions. We present calculation results in Section 3. In Section 3.1, we first confirm that our method can approximately reproduce the split-monopole force-free solution of slowly rotating Kerr BH magnetosphere, and then in Section 3.2, we perform calculations for the parabolic field model. We discuss the radial profiles of the density at the separation surface and compare our results with a mass loading mechanism and other studies in Section 4. Summary and prospects are presented in Section 5.

\section{MODEL}

To examine the density distribution inside the jet, we study the general relativistic equations for steady, axisymmetric, cold ideal MHD flows. We analytically solve the dynamics parallel to the prescribed poloidal field lines and numerically keep the transverse force balance at the separation surface.

The spacetime geometry is given by the Kerr metric in the Boyer-Lindquist coordinates,

$$
\begin{aligned}
d s^{2}= & -\left(1-\frac{2 r}{\Sigma}\right) d t^{2}-\frac{4 a r \sin ^{2} \theta}{\Sigma} d t d \phi+\frac{\Sigma}{\Delta} d r^{2} \\
& +\Sigma d \theta^{2}+\frac{\left[\left(r^{2}+a^{2}\right)^{2}-\Delta a^{2} \sin ^{2} \theta\right] \sin ^{2} \theta}{\Sigma} d \phi^{2},
\end{aligned}
$$

where $a$ is the dimensionless spin parameter, $\Sigma=r^{2}+$ $a^{2} \cos ^{2} \theta$, and $\Delta=r^{2}-2 r+a^{2}$. Hereafter we use the unit of $c=G=1$ and the $\mathrm{BH}$ mass $M=1$. The radius of the event horizon is $r_{\mathrm{H}}=1+\sqrt{1-a^{2}}$, and the angular velocity of the $\mathrm{BH}$ is $\omega_{\mathrm{H}}=a / 2 r_{\mathrm{H}}$.

\subsection{Bernoulli equation}

The basic equations are the energy-momentum equation, the Maxwell equations, and the mass flux conservation law with the steady, axisymmetric, ideal MHD conditions. They lead to the four integral constants along a field line (i.e. the Bernoulli constants), which are the total energy flux per particle $E$, the total angular momentum flux per particle $L$, the number density flux per magnetic flux $\eta$, and the so-called the 'angular velocity of the magnetic field' $\Omega_{\mathrm{F}}$ (Bekenstein \& Oron 1978). They are given as follows:

$$
\hat{E}(\Psi)=-u_{0}-\frac{\Omega_{\mathrm{F}} B_{3}}{4 \pi \mu \eta},
$$

$$
\begin{aligned}
\hat{L}(\Psi) & =u_{3}-\frac{B_{3}}{4 \pi \mu \eta}, \\
\eta(\Psi) & =-\frac{n u_{1}}{B_{1}} G_{\mathrm{t}}=-\frac{n u_{2}}{B_{2}} G_{\mathrm{t}}, \\
\Omega_{\mathrm{F}}(\Psi) & =\frac{F_{01}}{F_{13}}=\frac{F_{02}}{F_{23}} .
\end{aligned}
$$

Here we have introduced the magnetic flux function $\Psi(r, \theta)$ and the electromagnetic tensor $F_{\mu \nu}$. For the axisymmetric field, $F_{\mathrm{A} 3}=\partial_{\mathrm{A}} \Psi(A=1,2)$. Then the magnetic field is given by $B_{\mu}=1 / 2 \epsilon_{\nu \mu \lambda \sigma} \xi^{\nu} F^{\lambda \sigma}$, where $\xi^{\mu}=(1,0,0,0)$ is the time-like Killing vector, $\epsilon_{\mu \nu \lambda \sigma}=\sqrt{-g}[\mu \nu \lambda \sigma],[\mu \nu \lambda \sigma]$ is the permutation symbol, and $g \equiv \operatorname{det}\left(g_{\mu \nu}\right) . \quad F_{\mathrm{A} 0}=E_{\mathrm{A}}$ is the electric field. $n$ is the fluid-frame number density of the matter, $u^{\mu}$ is the four-velocity, and $\mu$ is the specific enthalpy. In the cold limit, $\mu$ is the rest energy of the plasma particle. $\hat{E}=E / \mu, \hat{L}=L / \mu$, and $G_{\mathrm{t}}=g_{00}+\Omega_{\mathrm{F}} g_{03}$.

Combination of Equations (2), (3), (4), and (5) is reduced to the Bernoulli equation, i.e., a fourth-order equation of the poloidal velocity $u_{\mathrm{p}}=\sqrt{u^{1} u_{1}+u^{2} u_{2}}$,

$$
\sum_{i=0}^{4} A_{i} u_{\mathrm{p}}^{i}=0
$$

where

$$
\begin{aligned}
& A_{4}=1, \\
& A_{3}=\frac{k_{0} B_{\mathrm{p}}}{2 \pi \mu \eta G_{\mathrm{t}}}, \\
& A_{2}=1+\hat{E}^{2} k_{4}+\left(\frac{k_{0} B_{\mathrm{p}}}{4 \pi \mu \eta G_{\mathrm{t}}}\right)^{2}, \\
& A_{1}=\frac{B_{\mathrm{p}}\left(k_{0}-\hat{E}^{2} k_{2}\right)}{2 \pi \mu \eta G_{\mathrm{t}}}, \\
& A_{0}=k_{0}\left(k_{0}-\hat{E}^{2} k_{2}\right)\left(\frac{B_{\mathrm{p}}}{4 \pi \mu \eta G_{\mathrm{t}}}\right)^{2} .
\end{aligned}
$$

Here, $k_{0}=-\left(g_{00}+2 \Omega_{\mathrm{F}} g_{03}+\Omega_{\mathrm{F}} g_{33}\right), k_{2}=\left(1-\hat{L} \Omega_{\mathrm{F}} / \hat{E}\right)^{2}$, $k_{4}=\left[g_{33}+2 g_{03} \hat{L} / \hat{E}+g_{00}(\hat{L} / \hat{E})^{2}\right] /\left(g_{03}^{2}-g_{00} g_{33}\right)$, and $B_{\mathrm{p}}=\sqrt{B_{1} B^{1}+B_{2} B^{2}}$ is the poloidal magnetic field. Equation (6) is identical with Equation (34) in $\mathrm{Pu}$ et al. (2015) and Equation (23) in Huang et al. (2019).

Given the integral constants $\left\{\hat{E}(\Psi), \hat{L}(\Psi), \eta(\Psi), \Omega_{\mathrm{F}}(\Psi)\right\}$ and the field line configuration $\Psi(r, \theta)$, one can solve the Bernoulli equation. Then one can derive the number density distribution along the field line from the definition of $\eta$ (Equation 4). The toroidal field can be calculated by

$$
B_{3}=-4 \pi \mu \eta \frac{G_{\phi} \hat{E}+G_{\mathrm{t}} \hat{L}}{M^{2}-k_{0}},
$$

with the Alfven Mach number $M^{2}=\pi \mu \eta^{2} / n$ and $G_{\phi}=$ $g_{03}+\Omega_{\mathrm{F}} g_{33}$. 


\begin{tabular}{l|rrrrrr} 
model & $\nu$ & $\epsilon$ & $a$ & $\Omega_{\mathrm{F}}(\Psi=1)$ & $u_{\mathrm{p}, \mathrm{ss}}$ & $\hat{E}_{0}$ \\
\hline M0 & 0 & 0 & 0.1 & $0.4999 \Omega_{\mathrm{H}}$ & $10^{-3}$ & $10^{3}$ \\
\hline P1 & 1 & $10^{-4}$ & 0.9 & $0.35 \Omega_{\mathrm{H}}$ & $10^{-3}$ & - \\
P2 & 1 & $10^{-4}$ & 0.8 & $0.35 \Omega_{\mathrm{H}}$ & $10^{-3}$ & - \\
P3 & 1 & $10^{-4}$ & 0.95 & $0.35 \Omega_{\mathrm{H}}$ & $10^{-3}$ & - \\
P4 & 1 & $10^{-4}$ & 0.9 & $0.35 \Omega_{\mathrm{H}}$ & $6 \times 10^{-4}$ & - \\
P5 & 1 & $10^{-4}$ & 0.9 & $0.35 \Omega_{\mathrm{H}}$ & $1.4 \times 10^{-3}$ & -
\end{tabular}

Table 1. Parameter values used in our models.

\subsection{Flux function model}

We assume that $\Psi(r, \theta)$ has a form of

$$
\Psi(r, \theta)=C\left[\left(\frac{r}{r_{\mathrm{H}}}\right)^{\nu}(1-\cos \theta)+\frac{1}{4} \epsilon r \sin \theta\right],
$$

where $C$ is the normalization factor setting $\Psi\left(r_{\mathrm{H}}, \pi / 2\right)=$ 1. $\nu$ and $\epsilon$ are the model parameters controlling the poloidal field line shape. For $\epsilon=0$, the field line configurations of $\nu=0$ and $\nu=1$ have the monopole and parabolic shape in the far zone, respectively. $\Psi(r, \theta)$ with $\nu=0$ and $\epsilon=0$ is the exact solution of the force-free magnetosphere in a Schwartzschild spacetime (Blandford \& Znajek 1977), and $\Psi(r, \theta)$ with $\nu=1$ and $\epsilon=0$ is the dominant term of the exact solution of the force-free magnetosphere in a Schwarzschild spacetime (Blandford \& Znajek 1977; Lee \& Park 2004). The jet-disk boundary in GRMHD simulations defined as the magnetic-to-matter energy flux ratio $\sigma=1-$ $\Omega_{\mathrm{F}} B_{3} /\left(4 \pi \mu \eta u_{0}\right) \mid=1$ matches the parabolic configurations with a constant $\nu$ in a large computational domain (Nakamura et al. 2018; Porth et al. 2019). $\epsilon$ represents a small disturbance from the force-free field lines, which makes the outflow accelerate by converting the Poynting flux to the kinetic energy flux (c.f. Beskin \& Nokhrina 2006; Pu et al. 2015). When $\epsilon=0, B_{\mathrm{p}} r^{2} \sin ^{2} \theta$ becomes constant along the field line in a far zone $(\mathrm{Pu} \&$ Takahashi 2020). For the outflow to pass through the fast magnetosonic point, $B_{\mathrm{p}} r^{2} \sin ^{2} \theta$ needs to decrease with the radius around the fast magnetosonic point (Begelman \& Li 1994; Beskin 2010; Toma \& Takahara 2013). When $\epsilon>0$, the field lines are collimated and $B_{\mathrm{p}} r^{2} \sin ^{2} \theta$ decreases. Then, the outflow can pass through the fast magnetosonic point.

In this paper, we consider two models, a splitmonopole configuration model $(\nu=0, \epsilon=0)$, and a perturbed parabolic configuration model $\left(\nu=1, \epsilon=10^{-4}\right)$. The aim of performing the split-monopole model is to check if our method can approximately reproduce the split-monopole force-free solution of slowly rotating Kerr BH magnetosphere, in which $\Omega_{\mathrm{F}} \approx 0.5 \Omega_{\mathrm{H}}$ and $\hat{E}(\Psi) \propto \sin ^{2} \theta$ (Blandford \& Znajek 1977). We set $a=0.1$ because such a small $\mathrm{BH}$ spin does not change
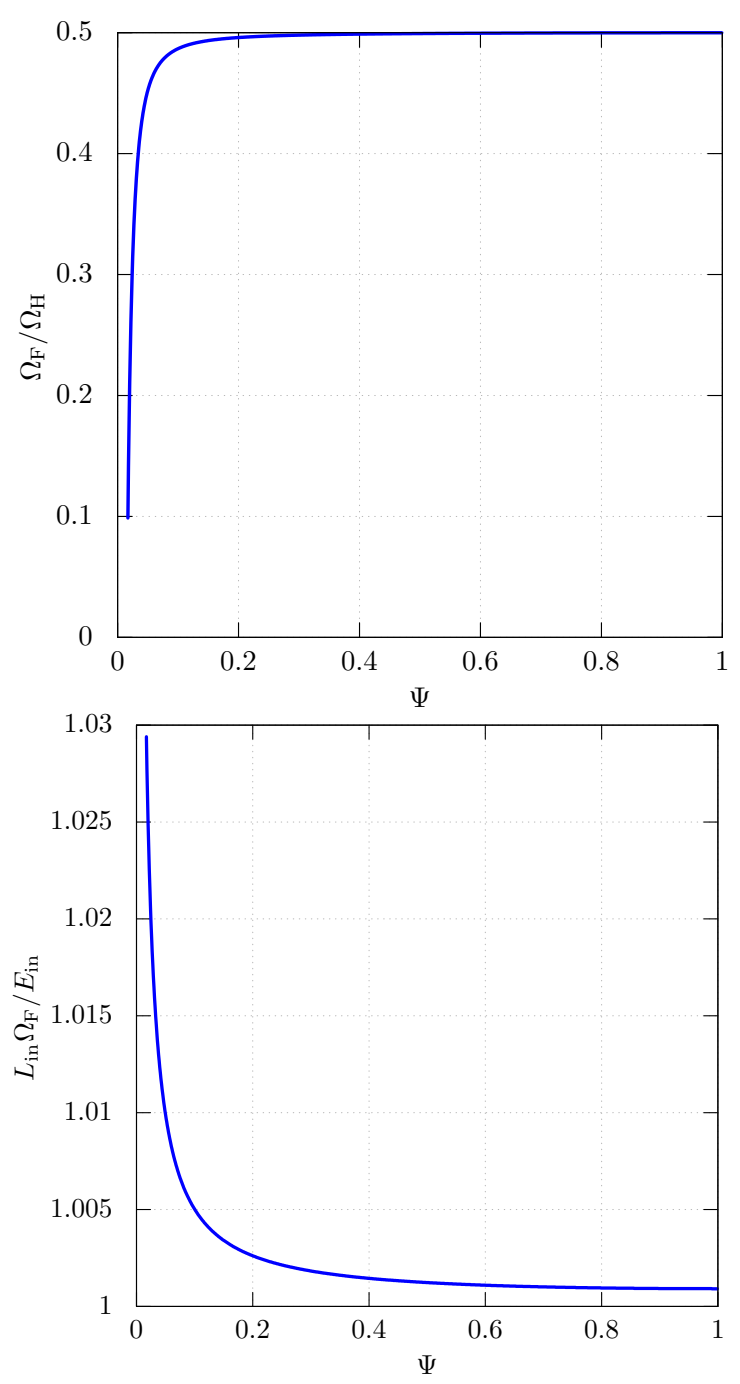

Figure 1. $\Omega_{\mathrm{F}}(\Psi)$ and $L_{\text {in }} \Omega_{\mathrm{F}} / E_{\text {in }}(\Psi)$ of the M0 model. Our result satisfies the conditions of the force-free split-monopole magnetosphere, $\Omega_{\mathrm{F}}=0.5 \Omega_{\mathrm{H}}$ and $L \Omega_{\mathrm{F}} / E=1$, within $1 \%$ accuracy in $\Psi>0.1$.

the field line structure significantly from the monopole force-free solution near the horizon (Tanabe \& Nagataki 2008; Tchekhovskoy et al. 2010). After confirming that our model can reproduce the split-monopole analytic solution well, we perform a calculation of a perturbed parabolic magnetosphere around a rapidly rotating $\mathrm{BH}$ $(a=0.9)$ to investigate the density distribution in the jet.

We list the parameter values used in our models in Table 1. The M0 model is the split-monopole configuration model, and the results are shown in Section 3.1. The P1 model is our fiducial model in the parabolic configuration models. We analyze the details of the P1 model in Section 3.2. The other parabolic configuration models (P2, P3, P4, and P5) are also analyzed to investigate the parameter dependence. 

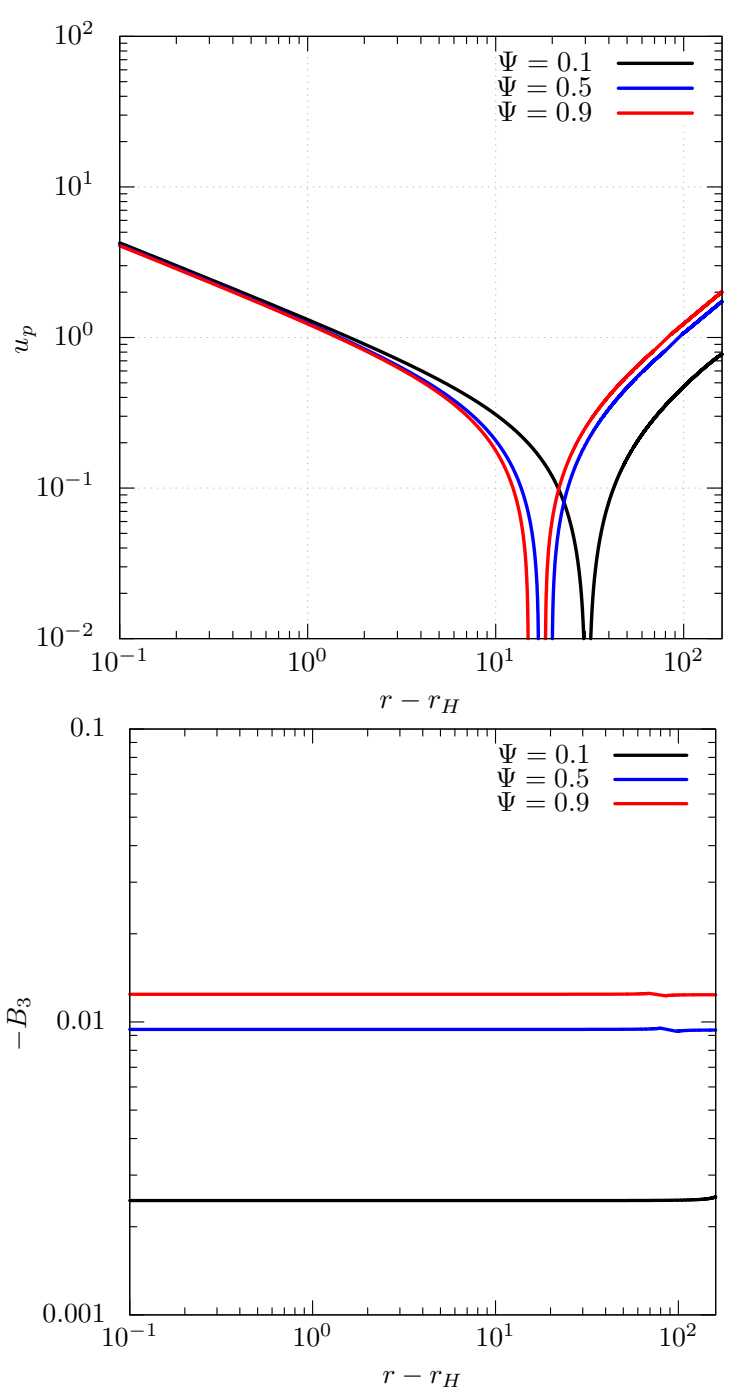

Figure 2. $u_{\mathrm{p}}, B_{3}$ along the field lines $\Psi=0.1,0.5$, and 0.9 of the split-monopole configuration model. We note that $u_{\mathrm{p}}$ is the absolute value of the poloidal velocity. The constant $B_{3}$ along the field line represents that the energy conversion from the Poynting flux to the kinetic energy flux is inefficient in this model.

\subsection{Determining the Bernoulli parameters}

Solving the wind equation (Equation 6) requires the Bernoulli parameters $\left\{\hat{E}(\Psi), \hat{L}(\Psi), \Omega_{\mathrm{F}}(\Psi), \eta(\Psi)\right\}$. We determine $\hat{L}(\Psi), \eta(\Psi)$, and $\Omega_{\mathrm{F}}(\Psi)$ in the same method for both of the field line configuration models, and determine $\hat{E}(\Psi)$ differently.

$\hat{L}(\Psi)$ and $\eta(\Psi)$ are determined simultaneously from the assumed poloidal velocity at the separation surface and the Znajek condition (Znajek 1977),

$$
\begin{aligned}
& u_{\mathrm{p}}\left(r_{\mathrm{ss}}, \theta\right)=u_{\mathrm{p}, \mathrm{ss}}=\text { const. } \\
& B_{3}\left(r_{\mathrm{H}}, \theta\right)=-\left[\left(\frac{g_{33}}{g_{22}}\right)^{1 / 2}\left(\omega_{\mathrm{H}}-\Omega_{\mathrm{F}}\right) \partial_{\theta} \Psi\right]_{\mathrm{r}=\mathrm{r}_{\mathrm{H}}}
\end{aligned}
$$

where $r_{\mathrm{ss}}$ denotes the radius of the separation surface. Combining $u_{\mathrm{p}, \mathrm{ss}}$ and the Znajek condition with Equation (6) at the separation surface and Equation (8) at the horizon, one can obtain the two equations for solving $\hat{L}$ and $\eta$. We set $u_{\mathrm{p}, \mathrm{ss}}=10^{-3}$ for the M0, P1, P2, and P3 model. The P2 and P3 models are used to investigate the $\mathrm{BH}$ spin dependence, while the $\mathrm{P} 4$ and $\mathrm{P} 5$ models are used to investigate the $u_{\mathrm{p}, \mathrm{ss}}$ dependence. The separation surface is defined by $\partial_{\mathrm{p}} k_{0}=0$, where $\partial_{\mathrm{p}} \equiv B^{1} \partial_{1}+B^{2} \partial_{2}$ is the differentiation in the direction along the field line.

$\Omega_{\mathrm{F}}(\Psi)$ is determined to satisfy the force balance between field lines at the separation surface. The component of the equation of motion perpendicular to the field line is

$$
e_{(\mathrm{n}) \mathrm{A}}\left[\rho u^{\nu} u_{; \nu}^{A}-F^{A \nu} I_{\nu}\right]=0,
$$

where $e_{(\mathrm{n}) \mathrm{A}}$ is the unit vector in the direction perpendicular to the field line in the poloidal plane $e_{(\mathrm{n}) \mathrm{A}}=$ $E_{\mathrm{A}} / \sqrt{E^{B} E_{\mathrm{B}}}(A, B=1,2)$. We assume $\Omega_{\mathrm{F}}(\Psi=1)=$ $0.4999 \Omega_{\mathrm{H}}$ for the M0 model, and $\Omega_{\mathrm{F}}(\Psi=1)=0.35 \Omega_{\mathrm{H}}$ for the parabolic configuration models. Then we choose $\Omega_{\mathrm{F}}(\Psi)$ by minimizing the left-hand side of Equation (12). We do this only at the separation surface to obtain the approximate solutions. We confirm that this method works well for the M0 model and then apply it to the parabolic configuration models. We also evaluate how well our approximate solutions keep the transverse balance at the regions other than the separation surface.

Regarding of $\hat{E}(\Psi)$, we take different treatments for the split-monopole and parabolic cases.

(i): For the M0 model, we set $\hat{E}=\hat{E}_{0} \sin ^{2} \theta$ and $\hat{E}_{0}=$ $10^{3}$, following the dependence of the Poynting flux in the force-free monopole magnetosphere (Blandford \& Znajek 1977). The small value of $u_{\mathrm{p}, \mathrm{ss}}$ is taken for the flow to be Poynting-flux dominated.

(ii): For the parabolic configuration models, we determine $\hat{E}$ for the outflow to accelerate smoothly passing through the fast magnetosonic point. If $\hat{E}$ is too low, $u_{\mathrm{p}}$ diverges after passing the Alfven point, while if $\hat{E}$ is too large, $u_{\mathrm{p}}$ does not diverge, but $M^{2}$ does not reach $M_{\text {fast }}^{2} \equiv k_{0}+$ $\left(G_{\mathrm{t}}^{2} B_{3}^{2}\right) /\left(\rho_{\mathrm{w}}^{2} B_{\mathrm{p}}^{2}\right)$, where $M^{2}=M_{\text {fast }}^{2}$ should be satisfied at the fast magnetosonic point (Takahashi et al. 1990). We adjust $\hat{E}$ so that $u_{\mathrm{p}}$ does not diverge and there is a point where $\left|1-M^{2} / M_{\text {fast }}^{2}\right|<$ $10^{-3}$.

We iteratively adjust $\hat{E}$ and $\Omega_{\mathrm{F}}$ to obtain their values satisfying both of the condition for smooth acceleration and the force balance at an expected precision. 
From Equation (4), $\eta$ has the opposite sign for the inflow and outflow. The sign of the second term of Equation (2) and (3) change between the inflow and outflow. On the other hand, the sign of the first term $u_{0}$ does not change, and $u_{0}$ is almost -1 at the separation surface. Due to this, $\hat{E}$ and $\hat{L}$ must have different values for the inflow and outflow to keep the electromagnetic field smooth and continuous at the separation surface. Thus, we set $\hat{E}_{\text {in }}=-\hat{E}_{\text {out }}+2$, where $\hat{E}_{\text {in }}$ and $\hat{E}_{\text {out }}$ are the values for inflow and outflow, respectively.

\section{RESULTS}

In Section 3.1, we perform the split-monopole configuration model and confirm that our model, which takes the trans-field balance only at the separation surface, can make the electromagnetic field consistent with the force-free solution. In Section 3.2, we perform the parabolic configuration model and show the density distribution inside the jet.

\subsection{Split-monopole configuration model}

Figure 1 shows $\Omega_{\mathrm{F}}(\Psi)$ and $\hat{L}_{\text {out }} \Omega_{\mathrm{F}} / \hat{E}_{\text {out }}(\Psi)$ at the separation surface. $\Omega_{\mathrm{F}} / \Omega_{\mathrm{H}}=0.5$ and the force-free condition $\hat{L}_{\text {out }} \Omega_{\mathrm{F}} / \hat{E}_{\text {out }}=1$ are satisfied within $1 \%$ accuracy in $\Psi>0.1$, which mean that our approximate solutions are consistent with the force-free monopole solution (Blandford \& Znajek 1977). The deviation from the monopole force-free solution decreases, as either the $\mathrm{BH}$ spin is smaller, $\hat{E}_{0}$ is larger, $u_{\mathrm{p}, \mathrm{ss}}$ is smaller, or $\Omega_{\mathrm{F}}(\Psi=1)$ is closer to $0.5 \Omega_{\mathrm{H}}$.

Figure 2 shows $u_{\mathrm{p}}$ and $B_{3}$ along the field lines of $\Psi=0.1,0.5,0.9$. The outflow does not pass through the fast magnetosonic point, unlike in the parabolic field configuration case, as discussed in Camenzind (1986). $B_{3}$ of each flow is almost constant along the field line unless it diverges. This means that the conversion from the Poynting flux to the fluid energy flux is inefficient in the monopole field configuration and that the electromagnetic field is almost force-free in the whole region.

\subsection{Parabolic configuration model}

\subsubsection{P1 model}

In this subsection, we focus on the results of the calculation of the P1 model. We show the two dimensional distribution of $u_{\mathrm{p}}, n$, and $\sigma$ in Figure 3. Here, we normalize the number density by

$$
n_{\text {norm }} \equiv\left[\frac{B_{1} B^{1}+B_{2} B^{2}+B_{3} B^{3}}{8 \pi \mu}\right]_{\left(\mathrm{r}=\mathrm{r}_{\mathrm{ss}}, \Psi=1\right)} .
$$

The inflow and outflow smoothly accelerate from the separation surface to relativistic speeds, and the density decreases with the distance from the separation surface.
We note that the density does not diverge at the separation surface since $u_{\mathrm{p}, \mathrm{ss}}$ is not zero. At the separation surface, $\sigma$ has the almost same value as $\hat{E}(\Psi)$ because $\left|u_{0}\right| \approx 1$ (see the top left panel of Figure 4). Along the separation surface, it increases from $\approx 10$ at the jet edge to the maximum value $\approx 40$, and then decreases to $\approx 10$ near the axis. Along the field line, $\sigma$ decreases as the radius increases for both the inflow and outflow.

Figure 4 shows $\eta(\Psi) E(\Psi), \hat{E}(\Psi), \Omega_{\mathrm{F}}(\Psi)$, and $n / n_{\text {norm }}$ at the separation surface. $\hat{E}(\Psi)$ has a peak at $\Psi \sim$ 0.3. The Poynting flux becomes zero at the axis, which means $\hat{E}(\Psi=0)=-u_{0} \approx 1$. $\Omega_{\mathrm{F}}$ increases toward $\Omega_{\mathrm{F}}=0.5 \Omega_{\mathrm{H}}$ from the edge to the axis, but it decreases near the axis at $\Psi \approx 0.25 . \hat{E} \approx \hat{L} \Omega_{\mathrm{F}}$ is satisfied within $1 \%$ accuracy. This means that the flow is Poynting flux dominated at the separation surface. $\eta E$ roughly follows $\propto \sin ^{2} \theta_{\mathrm{H}}$, while this dependence is that of $B_{3}$ on $\theta_{\mathrm{H}}$ at the horizon for $a \ll 1$ (Equation 11). The number density at the separation surface has the peak at the jet edge and decreases to nearly zero toward the jet axis.

Figure 5 shows $M^{2}, M_{\text {Alf }}^{2}$, and $M_{\text {fast }}^{2}$ along the field lines of $\Psi=0.1,0.5$, and 0.9 , where $M_{\text {Alf }}^{2} \equiv k_{0}$. The intersections of $M^{2}$ and $M_{\mathrm{Alf}}^{2}$ are the Alfven points, and the ones of $M^{2}$ and $M_{\text {fast }}^{2}$ are the fast magnetosonic points. Figure 6 shows $u_{\mathrm{p}}$ along the field lines $\Psi=0.1,0.5$, and 0.9. Both the inflow and outflow start from the separation surface and accelerate to relativistic speeds.

We evaluate the trans-field force-balance by introducing $\chi \equiv\left|\left(f_{+}-\left|f_{-}\right|\right) /\left(f_{+}+\left|f_{-}\right|\right)\right|$, where we gather the positive components of Equation (12) to $f_{+}$and the negative ones to $f_{-} . \quad \chi$ ranges from 0 to 1 , and $\chi=0$ means the complete force balance. The results show that $\chi$ is less than $10^{-6}$ for all the field lines at the separation surface. For the outflow above the separation surface, $\chi$ increases rapidly to $\sim 10^{-1}$ and then turns to decrease, while for the inflow, it increases up to $\sim 1$ and then decreases. The Lorentz force directs toward the axis $e_{(\mathrm{n}) \mathrm{A}} F^{A \nu} I_{\nu}>0$ and the inertial force directs in the opposite way $-e_{(\mathrm{n}) \mathrm{A}} \rho u^{A} u_{; \nu}^{\nu}<0$. These forces are well balanced at the separation surface $e_{(\mathrm{n}) \mathrm{A}} \rho u^{A} u_{; \nu}^{\nu} \approx e_{(\mathrm{n}) \mathrm{A}} F^{A \nu} I_{\nu}$. In other radii than the separation surface, the Lorentz force is larger than the inertial one, and the net force is directing for the flow to collimate.

\subsubsection{Parameter dependences}

We calculate the parabolic configuration models with different parameter values listed in Table 1 to investigate the dependencies of the density distribution on the $\mathrm{BH}$ spin $a$ and $u_{\mathrm{p}, \mathrm{ss}}$. 

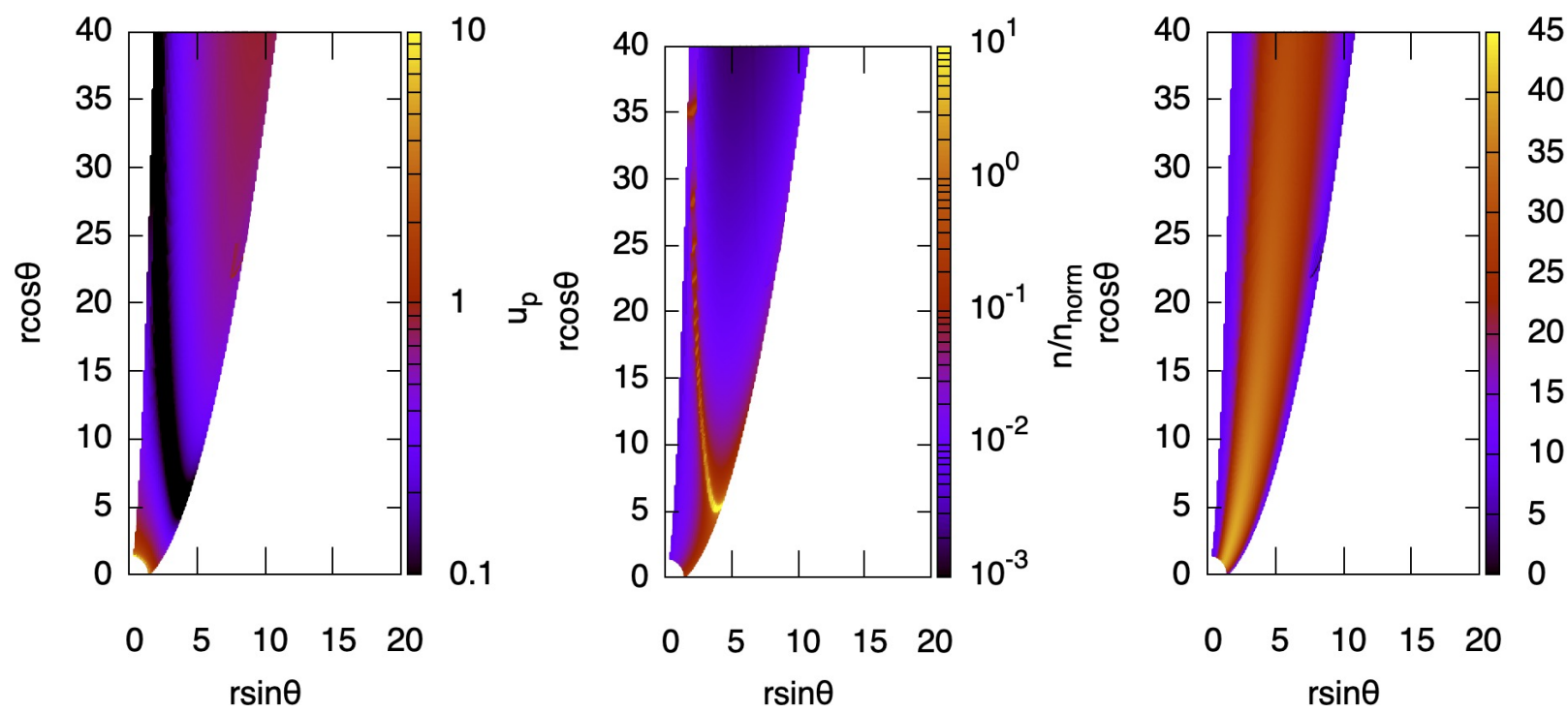

Figure 3. Two dimensional distribution of $u_{\mathrm{p}}, n / n_{\text {norm }}$, and $\sigma$ of the P1 model.

We use $a=0.8$ and 0.95 for the P2 and P3 models, respectively, to investigate the $\mathrm{BH}$ spin dependence. $\hat{E}, \Omega_{\mathrm{F}}, \eta E, n / n_{\text {norm }}$ are shown in Figure 4 . Interestingly, as $a$ becomes larger, the density gets larger near the jet edge and smaller near the axis, while $\hat{E}$ changes in the opposite way. $\eta E$ of the P2, P3 models also roughly follow $\propto \sin ^{2} \theta_{\mathrm{H}} . \eta E$ becomes larger in all the field lines with $a$ because of the increase of $B_{3}\left(r=r_{\mathrm{H}}\right)$ and the Poynting flux. $\Omega_{\mathrm{F}}$ also increases with $a$. The forcefreeness $1-L \Omega_{\mathrm{F}} / E$ is smaller than 0.2 for all three models. The minimum value $1-L \Omega_{\mathrm{F}} / E \approx 0.01$ realizes where $\hat{E}$ is maximum.

We perform calculations with different $u_{\mathrm{p}, \mathrm{ss}}$. We use $u_{\mathrm{p}, \mathrm{ss}}=6 \times 10^{-4}$ and $1.4 \times 10^{-3}$ for the $\mathrm{P} 4$ and $\mathrm{P} 5$ models, respectively. The results are shown in Figure 7. When $u_{\mathrm{p}, \mathrm{ss}}$ is smaller, $n / n_{\text {norm }}$ changes in a similar fashion as $a$ gets larger. $n / n_{\text {norm }}$ at the jet edge changes proportional to $u_{\mathrm{p}, \mathrm{ss}}^{-1}$. The $\mathrm{P} 1, \mathrm{P} 4$, and $\mathrm{P} 5$ models show that $\eta E$ does not significantly depend on $u_{\mathrm{p}, \mathrm{ss}}$. As $u_{\mathrm{p}, \mathrm{ss}}$ decreases, $\hat{E}$ increases and $\eta$ decreases for all the field lines.

\section{DISCUSSION}

\subsection{Density on the separation surface}

The matter density distribution will constrain the mass-loading mechanism. In Figure 8 , we show $n / n_{\text {norm }}$ of the P1, P2, and P3 models as a function of $r_{\mathrm{ss}}$. $n / n_{\text {norm }}$ is largest at the jet edge and decreases as $\Psi$ get smaller as shown in Figure 4. $r_{\mathrm{ss}}$ decreases as $a$ gets larger. At the far zone, the normalized density roughly follows $n / n_{\text {norm }} \propto r_{\mathrm{ss}}^{-2}$ in all the models. Figure 9 shows $n / n_{\text {norm }}$ as a function of $r_{\mathrm{ss}}$ for the different values of $u_{\mathrm{p}, \mathrm{ss}}$. We also have the dependence $n / n_{\text {norm }} \propto r_{\mathrm{ss}}^{-2}$ in the far zone in these models. For $r_{\mathrm{ss}}<10$, the $r_{\mathrm{ss}}$ dependence of $n / n_{\text {norm }}$ is steeper.

The annihilation of high-energy photons from the accretion disk is one of the proposed mass-loading mechanisms (Levinson \& Rieger 2011; Mościbrodzka et al. 2011; Kimura \& Toma 2020). This process leads to the $e^{+} e^{-}$density distribution $n \propto r^{-6}$ for the case in which $\gamma$-ray emitting region is compact near the BH (Mościbrodzka et al. 2011), and then the particles are injected mainly at the base for the outflow, i.e., at the separation surface, which is similar to the situation of our model. However, the dependence $r^{-6}$ appears too steep compared with the results in our model. If $\gamma$-ray emitting region is extended, say around $r \sim 10$, in the accretion flow (Kimura \& Toma 2020), the $r$ dependence of the $e^{+} e^{-}$density can be much shallower and might be consistent with our results. However, it should be noted that this $e^{+} e^{-}$injection model can provide particle number density sufficient for screening the spark gap (i.e., larger than the Goldreich-Julian number density), but not sufficient for the radio synchrotron flux of M87 jet (Kimura \& Toma 2020). Other injection mechanisms such as magnetic reconnection (e.g. Parfrey et al. 2015; Mahlmann et al. 2020) and/or fluid instability (e.g. Globus \& Levinson 2016; Nakamura et al. 2018; Chatterjee et al. 2019; Sironi et al. 2020) could be efficient for injecting electrons that produce the limbbrightened radio emission.

The results of our model are compatible with the observed limb-brightened emission structure of jets (see also Figure 3), although it is uncertain what fraction of the matter contribute to the non-thermal emission. The relative amount of the density near the axis compared to the one near the jet edge is also important because the 

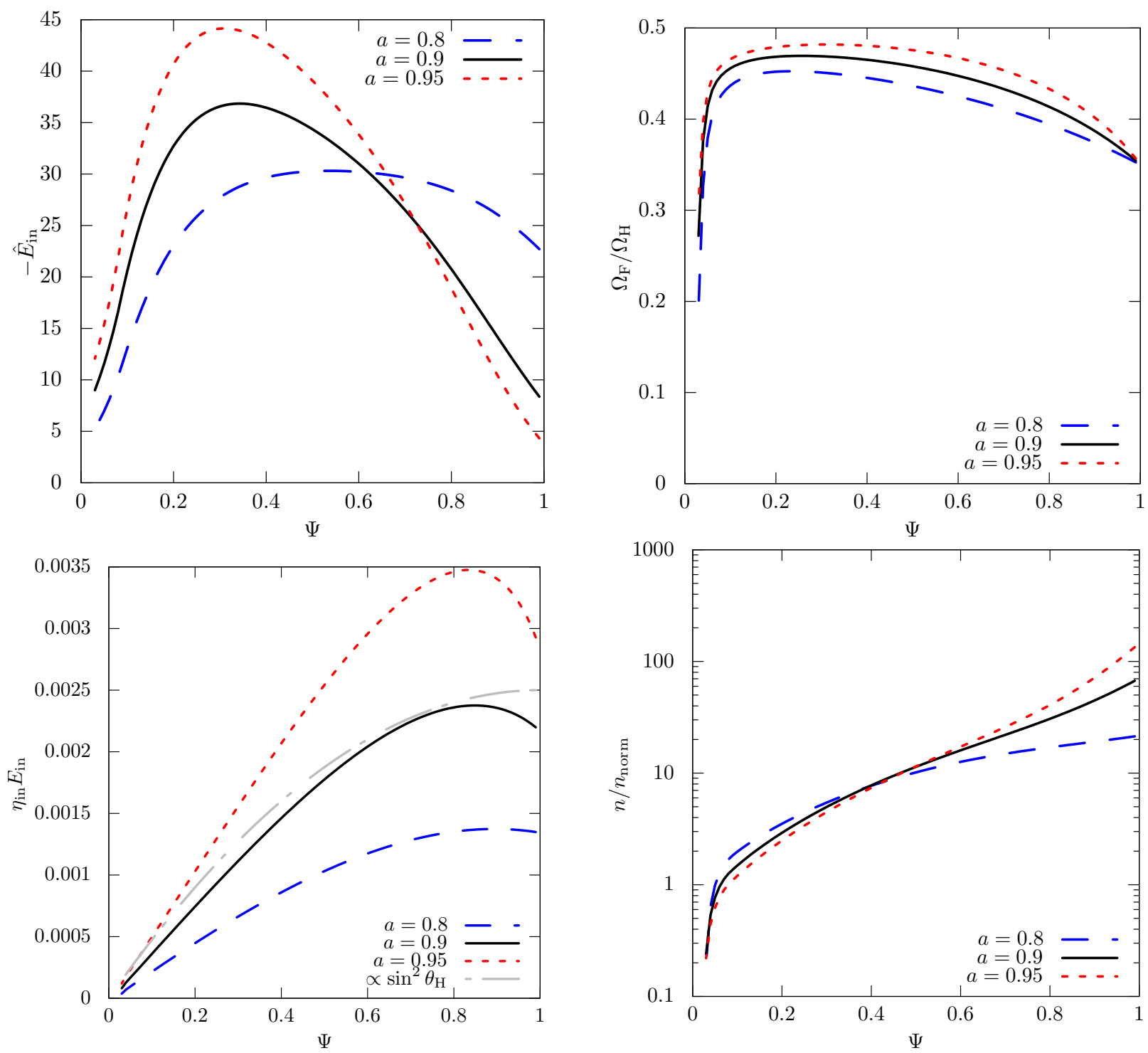

Figure 4. $\hat{E}_{\text {in }}, \Omega_{\mathrm{F}}, \eta_{\text {in }} E_{\text {in }}$, and $n / n_{\text {norm }}$ at the separation surface. The black, blue, and red lines are the results of P1, P2, and P3 models, respectively. The number density at the separation surface is concentrated more near the jet edge when the BH spin $a$ is larger.

emission near the axis will be Doppler-boosted, forming the central ridge of the observed triple-ridge emission structure of M87 jet (Ogihara et al. 2019).

\subsection{Comparison to other studies}

The distribution of the Bernoulli parameters can be compared to the results of other studies. Huang et al. (2020) numerically solved the GS equation for the whole region. They set the loading zone between the separation surface and the null-charge surface, although it is unclear whether such a large loading zone is necessary for obtaining the solutions. $\hat{E}(\Psi)$ has a peak near the jet edge in their result, while our models have the one relatively closer to the axis. They showed that $\Omega_{\mathrm{F}}$ monotonically increases toward the axis, and
$\Omega_{\mathrm{F}}(\Psi=0)=0.5 \Omega_{\mathrm{H}}$, which is set as a boundary condition, while in our model, $\Omega_{\mathrm{F}}(\Psi)$ decreases rapidly near the axis. $\eta$ of their model is assumed by a given magnetization parameter and the poloidal magnetic field at the null-charge surface. It is larger near the axis, which is the opposite trend from our results.

$\Omega_{\mathrm{F}}(\Psi)$ distribution is also shown in Beskin \& Zheltoukhov (2013), in which they solve the GS equation of a cylindrical jet in a special relativistic regime. In their result, $\Omega_{\mathrm{F}}(\Psi)$ decreases near the axis like our results. This trend is also seen in the GRMHD simulation of McKinney et al. (2012).

\subsection{Magnetic bending profile}



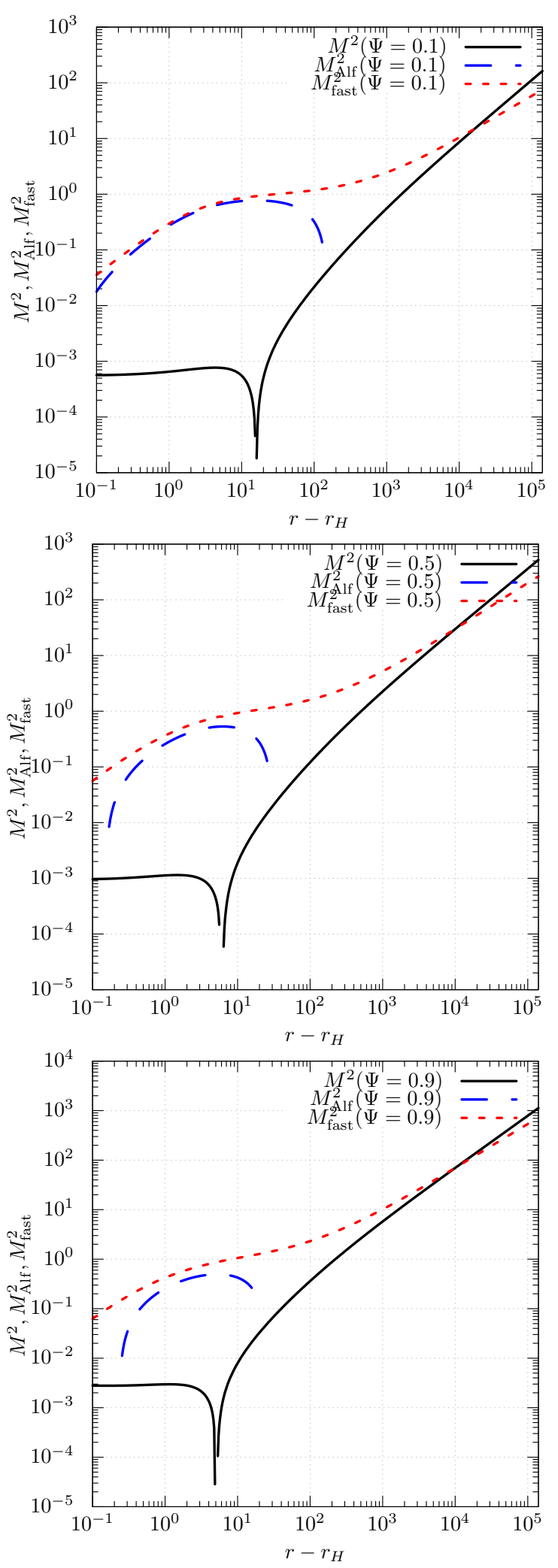

Figure 5. $M^{2}, M_{\text {Alf }}^{2}$, and $M_{\text {fast }}^{2}$ along the field lines of $\Psi=$ $0.1,0.5$, and 0.9 in the $\mathrm{P} 1$ model. The intersections of $M^{2}$ and $M_{\text {Alf }}^{2}$ are the Alfven points and the ones of $M^{2}$ and $M_{\text {fast }}^{2}$ are the fast magnetosonic points.

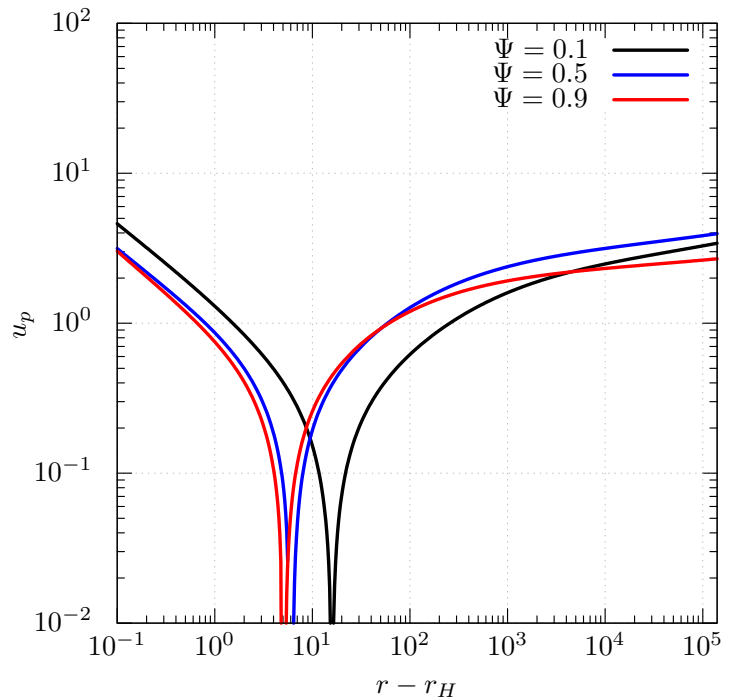

Figure 6. $u_{\mathrm{p}}$ along the field lines $\Psi=0.1,0.5$, and 0.9 in the P1 model. We note that $u_{\mathrm{p}}$ is the absolute value of the poloidal velocity.

$\mathrm{Pu}$ \& Takahashi (2020) introduced the reasonable shape of the function of $E_{\mathrm{p}, \mathrm{ZAMO}} / B_{\mathrm{T}, \mathrm{ZAMO}}$, which can be rewritten by the bending angle of the field line, and derived wind solutions with the prescribed function (see also Tomimatsu \& Takahashi 2003; Takahashi \& Tomimatsu 2008). $\quad E_{\mathrm{p}, \mathrm{ZAMO}}=\left|G_{\phi} B_{\mathrm{p}} /\left(G_{\mathrm{t}} \sqrt{g_{33}}\right)\right|$ is the poloidal electric field strength and $B_{\mathrm{T}, \mathrm{ZAMO}}=$ $\left|B_{3} /\left(\alpha \sqrt{g_{33}}\right)\right|$ is the toroidal magnetic field strength in the zero angular momentum observer frame.

There are some constraints to this function. First, $E_{\mathrm{p}, \mathrm{ZAMO}} / B_{\mathrm{T}, \mathrm{ZAMO}}=1$ at the horizon (i.e. the Znajek condition). Second, $E_{\mathrm{p}, \mathrm{ZAMO}} / B_{\mathrm{T}, \mathrm{ZAMO}}=0$ at the null-charge surface, where the field line corotate with the spacetime $\left(-g_{03} / g_{33}=\Omega_{\mathrm{F}}\right)$. Finally, $\left(E_{\mathrm{p}, \mathrm{ZAMO}} / B_{\mathrm{T}, \mathrm{ZAMO}}\right)^{2}<1-1 / \hat{E}^{2}$ for the outflow in order to prevent $u_{\mathrm{p}}$ from diverging. For the outflow, $E_{\mathrm{p}, \mathrm{ZAMO}} / B_{\mathrm{T}, \mathrm{ZAMO}}$ needs to increase for the flow to accelerate. Additionally, $E_{\mathrm{p}, \mathrm{ZAMO}} / B_{\mathrm{T}, \mathrm{ZAMO}}$ should be smooth and continuous.

The previous studies mentioned above prescribed $\left(E_{\mathrm{p}, \mathrm{ZAMO}} / B_{\mathrm{T}, \mathrm{ZAMO}}\right)^{2}$ as a constant value for outflow and derived $u_{\mathrm{p}}$ using it. The assumed $\left(E_{\mathrm{p}, \mathrm{ZAMO}} / B_{\mathrm{T}, \mathrm{ZAMO}}\right)^{2}$ and the derived one using Equation (8) was not self-consistent. We solved Equation (6) which does not explicitly depend on $B_{3}$, and derive $\left(E_{\mathrm{p}, \mathrm{ZAMO}} / B_{\mathrm{T}, \mathrm{ZAMO}}\right)^{2}$ afterwards. We show the self-consistent profile of $E_{\mathrm{p}, \mathrm{ZAMO}} / B_{\mathrm{T}, \mathrm{ZAMO}}$ of $\Psi=$ $0.1,0.5$, and 0.9 in Figure $10 .\left(E_{\mathrm{p}, \mathrm{ZAMO}} / B_{\mathrm{T}, \mathrm{ZAMO}}\right)^{2}$ in our result follows all the conditions listed above.

\subsection{Model limitations}



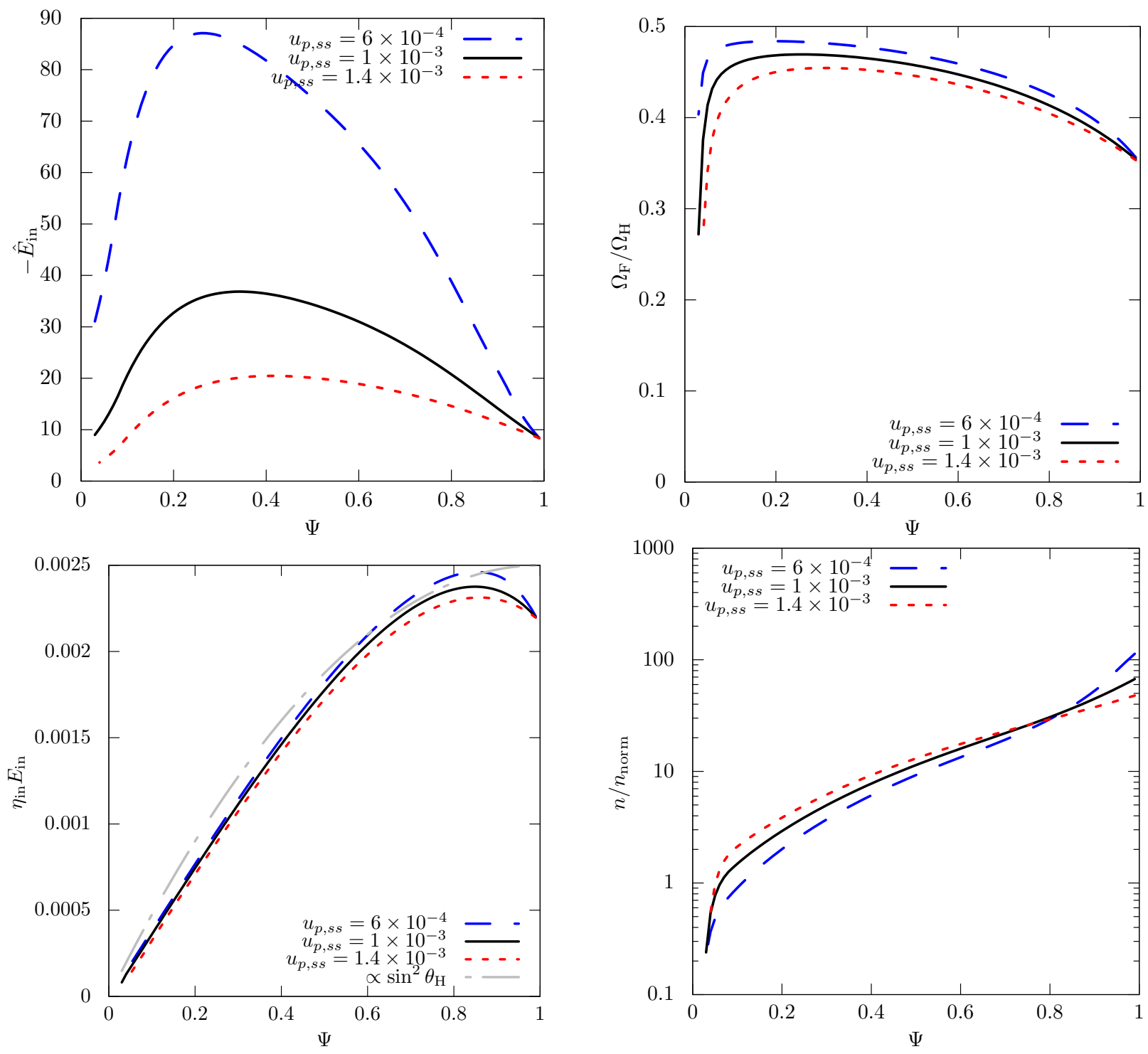

Figure 7. $\hat{E}_{\text {in }}, \Omega_{\mathrm{F}}, \eta_{\text {in }} E_{\text {in }}$, and $n / n_{\text {norm }}$ at the separation surface. The black, blue, and red lines are the results of P1, P4, and P5 models, respectively. The number density at the separation surface is concentrated more near the jet edge when $u_{\mathrm{p}, \mathrm{ss}}$ is smaller.

The inflows do not pass through the fast magnetosonic point in our results. The inflow diverges at the region very close to the horizon. Additionally, the indicator of the force-balance between the field lines $\chi$ is also large in the inflow region. We try to find different values of $\hat{E}_{\text {in }}$ from those satisfying the trans-field force-balance for the inflows to pass through the fast magnetosonic points, and find that $\left|\hat{E}_{\text {in }}\right|$ needs to be smaller only by a few in the case of the P1 model. The difference of $E_{\text {in }}$ is smaller for larger $\Psi$. Adjustment of $\Psi(r, \theta)$ should be considered in future work.

We note that if more particles are injected at regions closer to the $\mathrm{BH}$, like in the case of the annihilation of high-energy photons, the inflows are highly affected by mass loading and should be modeled with varying $\eta$ along a field line (cf. Globus \& Levinson 2014), while the current model of outflows is still applicable.

The distribution of $u_{\mathrm{p}, \mathrm{ss}}(\Psi)$ may differ between the injection mechanisms. In our model, we assume that $u_{\mathrm{p}, \mathrm{ss}}(\Psi)$ is constant for all the field line. If we change the distribution, it may change the density profile along the separation surface. This will be discussed in a separate work. Injection via instabilities and the magnetic reconnection may occur by the interaction between the jet edge and the disk wind (Globus \& Levinson 2016; Nakamura et al. 2018; Chatterjee et al. 2019; Sironi et al. 2020). This may change the field line configuration and the density distribution near the jet edge.

Although our current approximate solutions of GRMHD jets have the limitations mentioned above, 


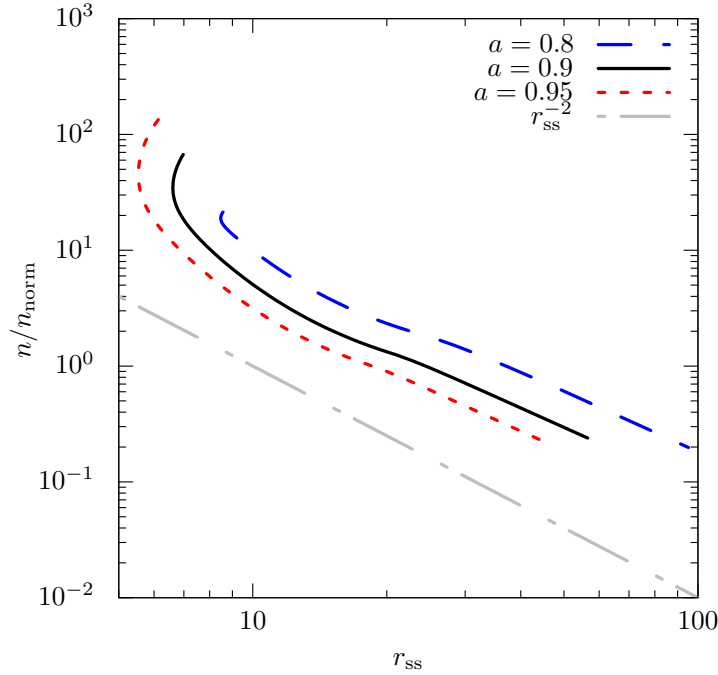

Figure 8. $n / n_{\text {norm }}$ as a function of $r_{\mathrm{ss}}$. The black, blue, and red lines are the results of the P1, P2, and P3 models, respectively. When the $\mathrm{BH}$ spin is larger, $r_{\mathrm{ss}}$ decreases. At the far zone, $n / n_{\text {norm }}$ roughly follows $\propto r_{\mathrm{ss}}^{-2}$.

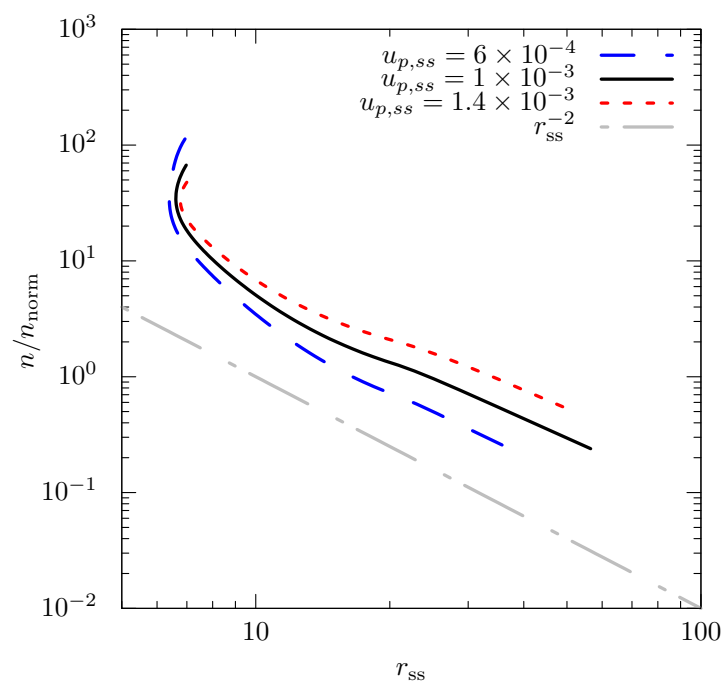

Figure 9. $n / n_{\text {norm }}$ as a function of $r_{\mathrm{ss}}$. The black, blue, and red lines are the results of the $\mathrm{P} 1, \mathrm{P} 4$, and $\mathrm{P} 5$ models, respectively. When the $\mathrm{BH}$ spin is larger, $r_{\mathrm{ss}}$ decreases. At the far zone, $n / n_{\text {norm }}$ roughly follows $\propto r_{\mathrm{ss}}^{-2}$.

they are useful not only for constraining the massloading mechanism but also for obtaining key ingredients from the observed complex emission structures. Indeed, by comparing the approximate special relativistic force-free steady jet models with observations of far-zone jets, it is found that the special relativistic beaming effect is essential for understanding the symmetry of the observed limb-brightened structure and the central ridge emission and that the emission image strongly depends on the matter density distribution at the base of outflow

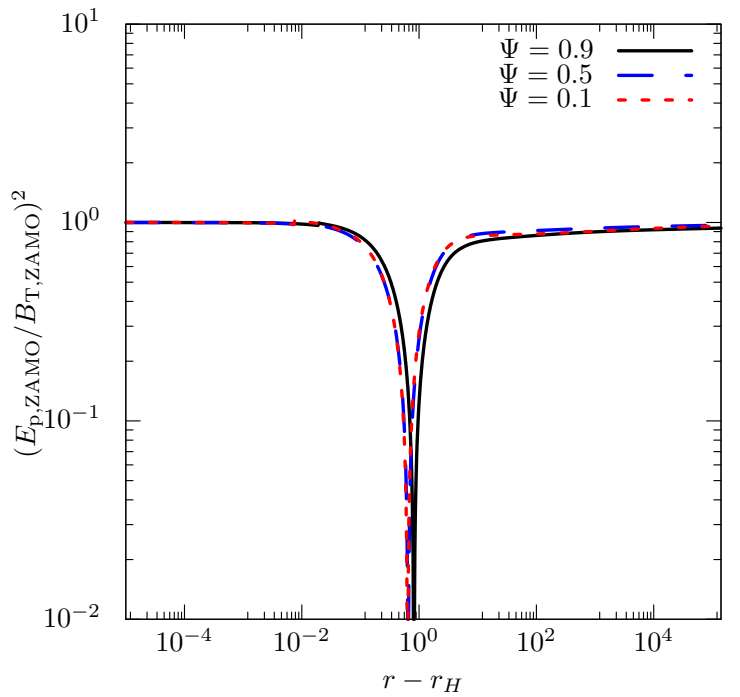

Figure 10. The radial profiles of $\left(E_{\mathrm{p}, \mathrm{ZAMO}} / B_{\mathrm{T}, \mathrm{ZAMO}}\right)^{2}$ of $\Psi=0.1,0.5$, and 0.9 in the $\mathrm{P} 1$ model. They are smooth and continuous and satisfy $\left(E_{\mathrm{p}, \mathrm{ZAMO}} / B_{\mathrm{T}, \mathrm{ZAMO}}\right)^{2}=1$ at the horizon from the Znajek condition, $\left(E_{\mathrm{p}, \mathrm{ZAMO}} / B_{\mathrm{T}, \mathrm{ZAMO}}\right)^{2}=$ 0 at the null-charge surface, and $\left(E_{\mathrm{p}, \mathrm{ZAMO}} / B_{\mathrm{T}, \mathrm{ZAMO}}\right)^{2}<$ $1-1 / \hat{E}^{2}$ in the outflow region.

(Takahashi et al. 2018; Ogihara et al. 2019). This has motivated us to develop GRMHD steady jet models.

\section{SUMMARY \& PROSPECTS}

We constructed a steady, axisymmetric GRMHD jet model to examine the density distribution inside the jet. We fixed the poloidal field line configuration, which mimics the ones of force-free or GRMHD simulation results with an additional term for obtaining trans-fastmagnetosonic outflows, and assumed the poloidal velocity of the flow at the separation surface $u_{\mathrm{p}, \mathrm{ss}}$ as constant for different field lines. We numerically solved the trans-field force-balance (Equation 12) at the separation surface to determine the Bernoulli parameters and analytically solved the fourth-order equation of the poloidal velocity along the field lines (Equation 6).

We consider the two field line configurations; (i) the split-monopole configuration model and (ii) the parabolic configuration model. (i) In the split-monopole configuration model, we confirmed that our method could reproduce the BZ solution well except around the axis. (ii) In the parabolic configuration model, we obtained approximate solutions of highly-magnetized GRMHD flows. The force-balance between field lines at the separation surface is satisfied with high accuracy. The outflow successfully passes the fast magnetosonic point and satisfies a good force-balance. We found that the number density distribution at the separation surface roughly scales as $n\left(r=r_{\mathrm{ss}}, \Psi\right) \propto r_{\mathrm{ss}}^{-2}$ in the far zone. We examined the parabolic configuration models 
with the different $\mathrm{BH}$ spins $a$ and $u_{\mathrm{p}, \mathrm{ss}}$. As the $\mathrm{BH}$ spin increases, the density at the separation surface is concentrated more at the jet edge. We obtained the similar trend when $u_{\mathrm{p}, \mathrm{ss}}$ is smaller.

Our semi-analytic model can be utilized to examine the density distribution of the highly magnetized jet region, while current GRMHD simulations cannot since they use the artificial density floor, and can survey a larger parameter space in detail because of the smaller computational budget. It will be interesting to apply radiative transfer calculations to our model and compare them to the observed emission structure, as done with the special relativistic force-free jet models (Takahashi et al. 2018; Ogihara et al. 2019).

Observations of core shifts, collimation profiles, proper motions of blobs, and Faraday rotation maps in various frequencies and scales (Kovalev et al. 2007; Asada et al. 2013; Hada et al. 2011, 2013, 2018; Nakamura \& Asada 2013; Mertens et al. 2016; Park et al. 2019; Park et al.
2019; Kim et al. 2020; Akiyama et al. 2018; Hiura et al. 2018; Giovannini et al. 2018; Kravchenko et al. 2020) will also be useful for testing jet models (Kino et al. 2014, 2015; Chael et al. 2019; Jeter et al. 2020). Future EHT and EATING-VLBI observations will provide crucial information on the jet launching region of M87 (Hada \& Eavn/Eating VLBI Collaboration 2020).

\section{ACKNOWLEDGMENTS}

We thank the anonymous referee for useful comments. We thank Shigeo Kimura, Hung-Yi Pu, Fumio Takahara, and Masaaki Takahashi for fruitful discussions. T.O. acknowledges financial support from the Graduate Program on Physics for the Universe of Tohoku University. This work is partly supported by Grant-in-Aid for JSPS Fellowship 20J14537 (T.O.) and KAKENHI No. $18 \mathrm{H} 01245$ (K.T.).

\section{REFERENCES}

Akiyama, K., Asada, K., Fish, V., et al. 2018, Galaxies, 6, 15, doi: 10.3390/galaxies6010015

Asada, K., Nakamura, M., Doi, A., Nagai, H., \& Inoue, M. 2013, The Astrophysical Journal, 781, L2, doi: $10.1088 / 2041-8205 / 781 / 1 / 12$

Asada, K., Nakamura, M., \& Pu, H.-Y. 2016, The Astrophysical Journal, 833, 56, doi: 10.3847/1538-4357/833/1/56

Begelman, M. C., \& Li, Z.-Y. 1994, ApJ, 426, 269, doi: 10.1086/174061

Bekenstein, J. D., \& Oron, E. 1978, Physical Review D, 18, 1809, doi: 10.1103/physrevd.18.1809

Beskin, V. S. 2010, MHD Flows in Compact Astrophysical Objects, doi: 10.1007/978-3-642-01290-7

Beskin, V. S., Istomin, Y. N., \& Parev, V. I. 1992, Soviet Astronomy, 36, 642

Beskin, V. S., \& Nokhrina, E. E. 2006, Monthly Notices of the Royal Astronomical Society, 367, 375, doi: 10.1111/j.1365-2966.2006.09957.x

Beskin, V. S., \& Zheltoukhov, A. A. 2013, Astronomy Letters, 39, 215, doi: 10.1134/s1063773713040014

Blandford, R. D., \& Znajek, R. L. 1977, Monthly Notices of the Royal Astronomical Society, 179, 433, doi: 10.1093/mnras/179.3.433

Boccardi, B., Krichbaum, T. P., Bach, U., Bremer, M., \& Zensus, J. A. 2016, Astronomy \& Astrophysics, 588, L9, doi: 10.1051/0004-6361/201628412

Broderick, A. E., \& Loeb, A. 2009, The Astrophysical Journal, 697, 1164, doi: 10.1088/0004-637x/697/2/1164
Broderick, A. E., \& Tchekhovskoy, A. 2015, The Astrophysical Journal, 809, 97, doi: 10.1088/0004-637x/809/1/97

Camenzind, M. 1986, A\&A, 162, 32

Chael, A., Narayan, R., \& Johnson, M. D. 2019, Monthly Notices of the Royal Astronomical Society, 486, 2873, doi: $10.1093 / \mathrm{mnras} / \mathrm{stz} 988$

Chael, A., Rowan, M. E., Narayan, R., Johnson, M. D., \& Sironi, L. 2018, doi: 10.1093/mnras/sty1261

Chatterjee, K., Liska, M., Tchekhovskoy, A., \& Markoff, S. B. 2019, MNRAS, 490, 2200, doi: $10.1093 / \mathrm{mnras} / \mathrm{stz} 2626$

Chatterjee, K., Younsi, Z., Liska, M., et al. 2020, MNRAS, 499, 362, doi: 10.1093/mnras/staa2718

Chernoglazov, A. V., Beskin, V. S., \& Pariev, V. I. 2019, Monthly Notices of the Royal Astronomical Society, 488, 224, doi: 10.1093/mnras/stz1683

Davelaar, J., Olivares, H., Porth, O., et al. 2019, Astronomy \& Astrophysics, 632, A2, doi: 10.1051/0004-6361/201936150

Dermer, C. D., \& Menon, G. 2009, High Energy Radiation from Black Holes: Gamma Rays, Cosmic Rays, and Neutrinos

Dexter, J. 2016, Monthly Notices of the Royal Astronomical Society, 462, 115, doi: 10.1093/mnras/stw1526

Dexter, J., McKinney, J. C., \& Agol, E. 2012, Monthly Notices of the Royal Astronomical Society, 421, 1517, doi: 10.1111/j.1365-2966.2012.20409.x 
Event Horizon Telescope Collaboration, Akiyama, K., Alberdi, A., et al. 2019a, ApJL, 875, L1, doi: $10.3847 / 2041-8213 /$ ab0ec7

—. 2019b, ApJL, 875, L2, doi: 10.3847/2041-8213/ab0c96

—. 2019c, ApJL, 875, L3, doi: 10.3847/2041-8213/ab0c57

—. 2019d, ApJL, 875, L4, doi: 10.3847/2041-8213/ab0e85

—. 2019e, ApJL, 875, L5, doi: 10.3847/2041-8213/ab0f43

—. 2019f, ApJL, 875, L6, doi: 10.3847/2041-8213/ab1141

Fendt, C. 1997, A\&A, 319, 1025

Giovannini, G., Savolainen, T., Orienti, M., et al. 2018, Nature Astronomy, 2, 472,

doi: 10.1038/s41550-018-0431-2

Globus, N., \& Levinson, A. 2014, The Astrophysical Journal, 796, 26, doi: 10.1088/0004-637x/796/1/26

Globus, N., \& Levinson, A. 2016, MNRAS, 461, 2605, doi: $10.1093 / \mathrm{mnras} / \mathrm{stw} 1474$

Hada, K. 2019, Galaxies, 8, 1, doi: 10.3390/galaxies8010001

Hada, K., Doi, A., Kino, M., et al. 2011, Nature, 477, 185, doi: 10.1038/nature10387

Hada, K., \& Eavn/Eating VLBI Collaboration. 2020, in Perseus in Sicily: From Black Hole to Cluster Outskirts, ed. K. Asada, E. de Gouveia Dal Pino, M. Giroletti, H. Nagai, \& R. Nemmen, Vol. 342, 73-76, doi: $10.1017 /$ S1743921318005173

Hada, K., Kino, M., Doi, A., et al. 2013, The Astrophysical Journal, 775, 70, doi: 10.1088/0004-637x/775/1/70

—. 2016, The Astrophysical Journal, 817, 131, doi: $10.3847 / 0004-637 x / 817 / 2 / 131$

Hada, K., Park, J. H., Kino, M., et al. 2017, Publications of the Astronomical Society of Japan, 69,

doi: $10.1093 / \mathrm{pasj} / \mathrm{psx} 054$

Hada, K., Doi, A., Wajima, K., et al. 2018, The Astrophysical Journal, 860, 141, doi: $10.3847 / 1538-4357 / \operatorname{aac} 49 f$

Hirotani, K., Pu, H.-Y., Lin, L. C.-C., et al. 2016, The Astrophysical Journal, 833, 142, doi: $10.3847 / 1538-4357 / 833 / 2 / 142$

Hiura, K., Nagai, H., Kino, M., et al. 2018, Publications of the Astronomical Society of Japan, 70,

doi: 10.1093/pasj/psy078

Huang, L., Pan, Z., \& Yu, C. 2019, The Astrophysical Journal, 880, 93, doi: 10.3847/1538-4357/ab2909

—. 2020, The Astrophysical Journal, 894, 45, doi: 10.3847/1538-4357/ab86a3

Jeter, B., Broderick, A. E., \& Gold, R. 2020, MNRAS, 493, 5606, doi: 10.1093/mnras/staa679

Jiménez-Rosales, A., \& Dexter, J. 2018, Monthly Notices of the Royal Astronomical Society, 478, 1875, doi: 10.1093/mnras/sty1210
Kawashima, T., Kino, M., \& Akiyama, K. 2019, The Astrophysical Journal, 878, 27, doi: $10.3847 / 1538-4357 /$ ab19c0

Kawashima, T., Toma, K., Kino, M., et al. 2020, arXiv e-prints, arXiv:2009.08641.

https://arxiv.org/abs/2009.08641

Kim, J.-Y., Krichbaum, T. P., Lu, R.-S., et al. 2018, Astronomy \& Astrophysics, 616, A188, doi: 10.1051/0004-6361/201832921

Kim, J.-Y., Krichbaum, T. P., Broderick, A. E., et al. 2020, A\&A, 640, A69, doi: 10.1051/0004-6361/202037493

Kimura, S. S., \& Toma, K. 2020, arXiv e-prints, arXiv:2003.13173. https://arxiv.org/abs/2003.13173

Kino, M., Takahara, F., Hada, K., et al. 2015, The Astrophysical Journal, 803, 30, doi: 10.1088/0004-637x/803/1/30

Kino, M., Takahara, F., Hada, K., \& Doi, A. 2014, The Astrophysical Journal, 786, 5, doi: $10.1088 / 0004-637 x / 786 / 1 / 5$

Kinoshita, S., \& Igata, T. 2018, Progress of Theoretical and Experimental Physics, 2018, 033E02, doi: $10.1093 /$ ptep/pty024

Kisaka, S., Levinson, A., \& Toma, K. 2020, The Astrophysical Journal, 902, 80, doi: $10.3847 / 1538-4357 /$ abb46c

Koide, S., Shibata, K., Kudoh, T., \& Meier, D. L. 2002, Science, 295, 1688, doi: 10.1126/science.1068240

Komissarov, S. S. 2004, Monthly Notices of the Royal Astronomical Society, 350, 427, doi: 10.1111/j.1365-2966.2004.07598.x

Komissarov, S. S., Barkov, M. V., Vlahakis, N., \& Königl, A. 2007, Monthly Notices of the Royal Astronomical Society, 380, 51, doi: 10.1111/j.1365-2966.2007.12050.x

Komissarov, S. S., Vlahakis, N., \& Königl, A. 2010, MNRAS, 407, 17, doi: 10.1111/j.1365-2966.2010.16779.x

Kovalev, Y. Y., Lister, M. L., Homan, D. C., \& Kellermann, K. I. 2007, The Astrophysical Journal, 668, L27, doi: 10.1086/522603

Kravchenko, E., Giroletti, M., Hada, K., et al. 2020, A\&A, 637, L6, doi: 10.1051/0004-6361/201937315

Lee, H., \& Park, J. 2004, Physical Review D, 70, doi: 10.1103/physrevd.70.063001

Levinson, A., \& Rieger, F. 2011, The Astrophysical Journal, 730, 123, doi: 10.1088/0004-637x/730/2/123

Levinson, A., \& Segev, N. 2017, Physical Review D, 96, doi: 10.1103/physrevd.96.123006

Lu, R.-S., Broderick, A. E., Baron, F., et al. 2014, The Astrophysical Journal, 788, 120, doi: $10.1088 / 0004-637 x / 788 / 2 / 120$ 
Lyubarsky, Y. E. 2009, Monthly Notices of the Royal Astronomical Society, 402, 353, doi: 10.1111/j.1365-2966.2009.15877.x

Mahlmann, J. F., Cerdá-Durán, P., \& Aloy, M. A. 2018, Monthly Notices of the Royal Astronomical Society, 477, 3927, doi: 10.1093/mnras/sty858

Mahlmann, J. F., Levinson, A., \& Aloy, M. A. 2020, MNRAS, 494, 4203, doi: 10.1093/mnras/staa943

McKinney, J. C., \& Blandford, R. D. 2009, Monthly Notices of the Royal Astronomical Society: Letters, 394, L126, doi: 10.1111/j.1745-3933.2009.00625.x

McKinney, J. C., \& Gammie, C. F. 2004, The Astrophysical Journal, 611, 977, doi: 10.1086/422244

McKinney, J. C., Tchekhovskoy, A., \& Blandford, R. D. 2012, Monthly Notices of the Royal Astronomical Society, 423, 3083, doi: 10.1111/j.1365-2966.2012.21074.x

Mertens, F., Lobanov, A. P., Walker, R. C., \& Hardee, P. E. 2016, Astronomy \& Astrophysics, 595, A54, doi: 10.1051/0004-6361/201628829

Mościbrodzka, M., Dexter, J., Davelaar, J., \& Falcke, H. 2017, Monthly Notices of the Royal Astronomical Society, 468, 2214, doi: 10.1093/mnras/stx587

Mościbrodzka, M., Falcke, H., \& Shiokawa, H. 2016, Astronomy \& Astrophysics, 586, A38, doi: 10.1051/0004-6361/201526630

Mościbrodzka, M., Falcke, H., Shiokawa, H., \& Gammie, C. F. 2014, Astronomy \& Astrophysics, 570, A7, doi: 10.1051/0004-6361/201424358

Mościbrodzka, M., Gammie, C. F., Dolence, J. C., \& Shiokawa, H. 2011, The Astrophysical Journal, 735, 9, doi: 10.1088/0004-637x/735/1/9

Nagai, H., Haga, T., Giovannini, G., et al. 2014, The Astrophysical Journal, 785, 53, doi: $10.1088 / 0004-637 x / 785 / 1 / 53$

Nakamura, M., \& Asada, K. 2013, The Astrophysical Journal, 775, 118, doi: 10.1088/0004-637x/775/2/118

Nakamura, M., Asada, K., Hada, K., et al. 2018, The Astrophysical Journal, 868, 146, doi: $10.3847 / 1538-4357 /$ aaeb2d

Nathanail, A., \& Contopoulos, I. 2014, The Astrophysical Journal, 788, 186, doi: 10.1088/0004-637x/788/2/186

Nitta, S.-y., Takahashi, M., \& Tomimatsu, A. 1991, Physical Review D, 44, 2295, doi: 10.1103/physrevd.44.2295

Ogihara, T., Takahashi, K., \& Toma, K. 2019, The Astrophysical Journal, 877, 19, doi: $10.3847 / 1538-4357 /$ ab1909

Pan, Z., Yu, C., \& Huang, L. 2017, The Astrophysical Journal, 836, 193, doi: 10.3847/1538-4357/aa5c36
Parfrey, K., Giannios, D., \& Beloborodov, A. M. 2015, Monthly Notices of the Royal Astronomical Society: Letters, 446, L61, doi: 10.1093/mnrasl/slu162

Park, J., Hada, K., Kino, M., et al. 2019, The Astrophysical Journal, 871, 257, doi: 10.3847/1538-4357/aaf9a9

Park, J., Hada, K., Kino, M., et al. 2019, ApJ, 887, 147, doi: 10.3847/1538-4357/ab5584

Piner, B. G., Pant, N., \& Edwards, P. G. 2010, The Astrophysical Journal, 723, 1150, doi: 10.1088/0004-637x/723/2/1150

Piner, B. G., Pant, N., Edwards, P. G., \& Wiik, K. 2008, The Astrophysical Journal, 690, L31, doi: 10.1088/0004-637x/690/1/131

Porth, O., Fendt, C., Meliani, Z., \& Vaidya, B. 2011, The Astrophysical Journal, 737, 42, doi: $10.1088 / 0004-637 x / 737 / 1 / 42$

Porth, O., Chatterjee, K., Narayan, R., et al. 2019, ApJS, 243, 26, doi: 10.3847/1538-4365/ab29fd

Pu, H.-Y., Nakamura, M., Hirotani, K., et al. 2015, The Astrophysical Journal, 801, 56, doi: 10.1088/0004-637x/801/1/56

Pu, H.-Y., \& Takahashi, M. 2020, The Astrophysical Journal, 892, 37, doi: 10.3847/1538-4357/ab77ab

Riordan, M. O., Pe'er, A., \& McKinney, J. C. 2018, The Astrophysical Journal, 853, 44, doi: 10.3847/1538-4357/aaa0c4

Sironi, L., Rowan, M. E., \& Narayan, R. 2020, arXiv e-prints, arXiv:2009.11877. https://arxiv.org/abs/2009.11877

Takahashi, H. R., Ohsuga, K., Kawashima, T., \& Sekiguchi, Y. 2016, The Astrophysical Journal, 826, 23, doi: $10.3847 / 0004-637 x / 826 / 1 / 23$

Takahashi, K., Toma, K., Kino, M., Nakamura, M., \& Hada, K. 2018, The Astrophysical Journal, 868, 82, doi: $10.3847 / 1538-4357 /$ aae832

Takahashi, M., Nitta, S., Tatematsu, Y., \& Tomimatsu, A. 1990, The Astrophysical Journal, 363, 206, doi: 10.1086/169331

Takahashi, M., \& Tomimatsu, A. 2008, Physical Review D, 78, doi: 10.1103/physrevd.78.023012

Tanabe, K., \& Nagataki, S. 2008, Physical Review D, 78, doi: $10.1103 /$ physrevd.78.024004

Tanaka, S. J., \& Toma, K. 2020, Monthly Notices of the Royal Astronomical Society, 494, 338, doi: $10.1093 / \mathrm{mnras} / \mathrm{staa} 728$

Tchekhovskoy, A., McKinney, J. C., \& Narayan, R. 2008, Monthly Notices of the Royal Astronomical Society, 388, 551, doi: 10.1111/j.1365-2966.2008.13425.x

—. 2009, The Astrophysical Journal, 699, 1789, doi: 10.1088/0004-637x/699/2/1789 
Tchekhovskoy, A., Narayan, R., \& McKinney, J. C. 2010, The Astrophysical Journal, 711, 50, doi: $10.1088 / 0004-637 x / 711 / 1 / 50$

—. 2011, Monthly Notices of the Royal Astronomical Society: Letters, 418, L79, doi: 10.1111/j.1745-3933.2011.01147.x

Toma, K., \& Takahara, F. 2013, Progress of Theoretical and Experimental Physics, 2013, 083E02, doi: $10.1093 /$ ptep/ptt058

-. 2014, Monthly Notices of the Royal Astronomical Society, 442, 2855, doi: 10.1093/mnras/stu1053
- 2016, Progress of Theoretical and Experimental Physics, 2016, 063E01, doi: 10.1093/ptep/ptw081

Tomimatsu, A., \& Takahashi, M. 2003, The Astrophysical Journal, 592, 321, doi: 10.1086/375579

Walker, R. C., Hardee, P. E., Davies, F. B., Ly, C., \& Junor, W. 2018, The Astrophysical Journal, 855, 128, doi: $10.3847 / 1538-4357 /$ aaafcc

Walker, R. C., Ly, C., Junor, W., \& Hardee, P. J. 2008, Journal of Physics: Conference Series, 131, 012053, doi: 10.1088/1742-6596/131/1/012053

Znajek, R. L. 1977, Monthly Notices of the Royal Astronomical Society, 179, 457, doi: 10.1093/mnras/179.3.457 